\title{
Optical Communications Research at Institute of Telecommunications
}

\section{J. F. ROCHA}

University of Aveiro, Aveiro, Portugal

\section{A. T. CARTAXO}

Instituto Superior Técnico

Torre Norte

Lisboa, Portugal

\section{H. J. SILVA}

University of Coimbra

Coimbra, Portugal

\section{J. L. PINTO}

A. L. TEIXEIRA

A. S. GAMEIRO

University of Aveiro, Aveiro, Portugal

\section{P. P. MONTEIRO}

University of Aveiro, Aveiro, Portugal, and Siemens S.A.

Amadora, Portugal

\section{J. O. PIRES}

Instituto Superior Técnico

Torre Norte

Lisboa, Portugal

R. S. RIBEIRO

M. O. VIOLAS

A. N. PINTO

M. N. LIMA

P. S. ANDRÉ

University of Aveiro, Aveiro, Portugal

\section{M. FREIRE}

University of Coimbra

Coimbra, Portugal, and

University of Beira Interior

Covilhã, Portugal

A. L. RIBEIRO

C. F. FERNANDES

J. T. PEREIRA

Instituto Superior Técnico

Torre Norte

Lisboa, Portugal

J. M. POUSA

J. M. SANTOS

M. T. ALMEIDA

University of Aveiro, Aveiro, Portugal, and PT Inovação, S.A.

Aveiro, Portugal

\section{A. R. TAVARES \\ R. N. NOGUEIRA}

University of Aveiro, Aveiro, Portugal

\section{J. S. MACHADO}

University of Aveiro, Aveiro, Portugal, and PT Inovação, S.A.

Aveiro, Portugal

Received 13 December 2004; accepted 28 February 2005.

Address correspondence to J. F. Rocha, Institute of Telecommunications, Pólo de Aveiro, University of Aveiro, Campus Universitário de Santiago, P-3810-193, Aveiro, Portugal. E-mail: frocha@ieee.org 
In this contribution we present a brief summary of research activities carried out at Instituto de Telecomunicações (IT, Institute of Telecommunications) in optical communications. These activities cover various aspects related with optical components/subsystems, optical communication systems, and optical networking.

Keywords optical communications, optical networks, optical communication systems, opto-electronic components

\section{Introduction}

Instituto de Telecomunicações (IT, Institute of Telecommunications) is a private, not-forprofit organization, of public interest, a partnership of four institutions with experience and tradition in research and development in the field of telecommunications: Instituto Superior Técnico (IST), Universidade de Aveiro (UA), Faculdade de Ciências e Tecnologia da Universidade de Coimbra (FCTUC), and Portugal Telecom Inovação, S.A. (PTIN). It was created with support of a 7.5 million Euro grant from CIÊNCIA Programme. This investment enabled the building of new infrastructures and equipping of research laboratories. Installation was concluded at Aveiro in 1993 and at Lisbon and Coimbra in 1994. Figure 1 shows part of the research facilities for optical communications at Aveiro. In recognition of its achievements, IT was awarded the status of Associate Laboratory of the Portuguese Ministry for Science and Technology in 2001. Recently, an external laboratory was created at the University of Beira Interior, Covilhã, and a delegation opened at the Instituto Politécnico de Leiria, Leiria. In 2004 Siemens Portugal S.A. also became a partner of IT.

The main research and education activities in IT are incorporated in the following research areas: wireless communications, optical communications, networks, and multimedia. These areas interact with a horizontal area of basic sciences (applied mathematics and physics, and communication theory) and support technologies for telecommunications (electronics, and instrumentation, and measurements). In this contribution, the ongoing research in optical communications will be described. Our activities aim at considering the implementation of optical networks in an integrated way. Consequently, our research covers various aspects related with components and subsystems, communication systems

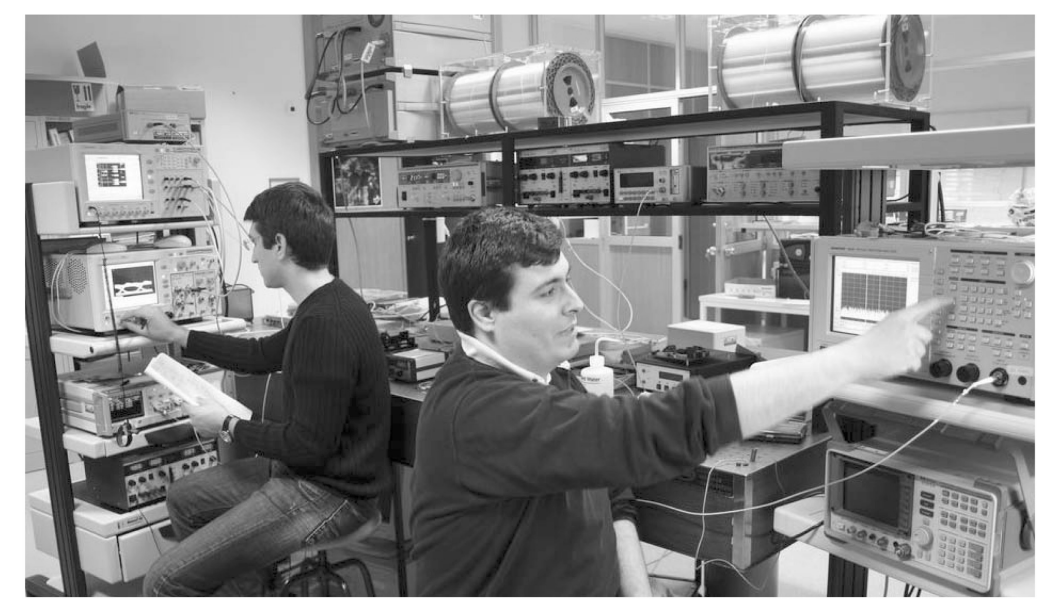

Figure 1. Partial view of optical communications research facilities at IT Aveiro. 
and networking, with most of the work geared toward the implementation of all-optical networks. In the following text, our main research results are grouped under headings that coincide with the three aspects of optical communications mentioned above.

\section{Optical Components and Subsystems}

In the field of optical components and subsystems, the following aspects are being considered:

- Modeling, analysis, optimization and implementation of optical devices, including optical sources [1, 2], detectors, fiber Bragg grating devices [3-8] and speciality fibers.

- Modeling, analysis/characterization, optimization, and implementation of subsystems. Work concentrates on optical add-drop multiplexers, optical crossconnects [9], routers, wavelength converters [10, 11], amplifiers [12], optical sources [13], optical receivers, all-optical gates, and signal processors. These subsystems are intended for bit rates of $40 \mathrm{Gbit} / \mathrm{s}$ and above, aiming at $160 \mathrm{Gbit} / \mathrm{s}$.

In the area of optical amplification, IT is optimizing Raman, doped, and semiconductor optical amplifiers. Regarding the first amplifier type, multipump configurations based on fiber Bragg grating filters are used to achieve equalized gain over wider amplification range. In relation to the erbium-doped fiber amplifiers, extended operation over the L-band is being explored in terms of experimental implementation and characterization, by developing and tuning amplifier models able to fit both the noise and the gain of practical amplifiers into simulation environments (Figure 2) [12]. Fiber/waveguide index and doping profile is optimized by improving the models for the amplification gain and behavior. In terms of semiconductor optical amplifiers its dynamic behavior is being studied and modeled in order to achieve a better matching with experimental results and also to explore further applications (as gating operation and others).

In the optical sources area we have developed a novel architecture for the selfgeneration of two independent pumps with orthogonal polarizations in a single optical ring cavity. It is based on two tunable fiber Bragg gratings, a gain medium (e.g., optical amplifier), and a polarization controller. The key elements are the two gratings that were written in high-birefringence fibers. In this way, they act as selective polarization and wavelength filters, essential for the orthogonal pump generation. With this simple
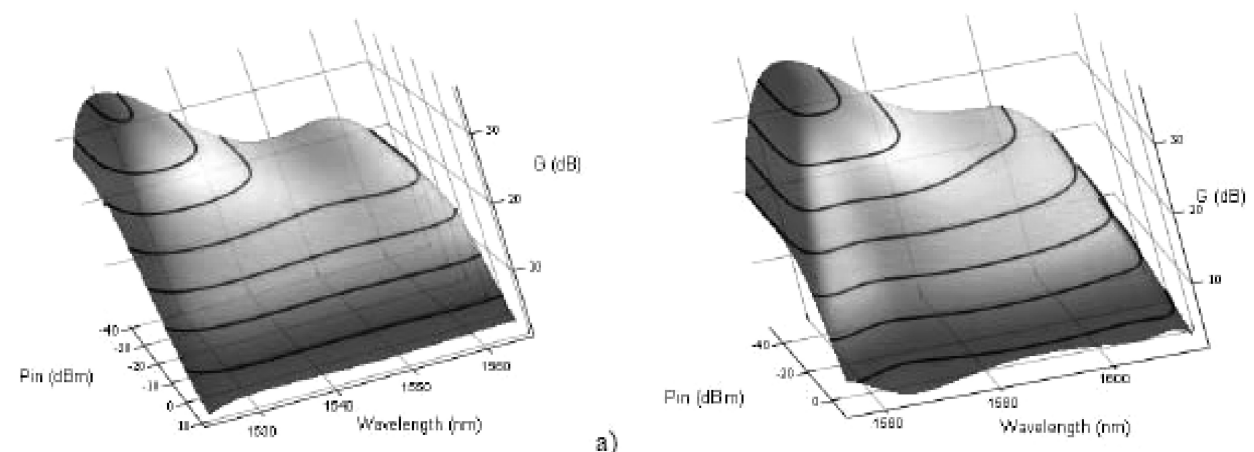

a)

Figure 2. 3D model representation of gain dependence on the input power and wavelength for EDFA working in C-band (a) and L-band (b). 
architecture, it is possible to self-generate two tunable pumps with stable power in a costeffective way. This dual pump architecture was used with a dispersion-shifted fiber (DSF) to implement a tunable broadband wavelength converter. The two pumps enable versatile conversion over a wide wavelength span. The results showed conversion efficiencies up to $-2 \mathrm{~dB}$ with a span $>15 \mathrm{~nm}$ and with the signal-to-noise ratio of the converted signal up to $26 \mathrm{~dB}$. The architecture enables a relatively low-cost implementation in comparison to other two-pump configurations, which need two external lasers to achieve a versatile tunable converter. We have also used this architecture for wavelength conversion based on FWM in a semiconductor optical amplifier (SOA) [14]. In this way, a tunable broadband wavelength converter with constant efficiency over the entire SOA gain band has been implemented in a very cost-effective architecture, since it only needs a small number of components. As it is based on four-wave mixing, the converter is also transparent to bit rate and signal format.

Another area of expertise in IT concerns the optimization and implementation of post-detection filters for multigigabit optical receivers. These filters have the main function of maximizing the signal-to-noise ratio at the input to the decision devices, while at the same time minimizing intersymbol interference (ISI) and providing tolerance to timing impairments from the signal and timing clock jitter. Different microwave and millimeter filter design strategies were investigated to be used at receiver units developed for the optical communication system demonstrators of the European Community ACTS (Advanced Communications Technologies and Services) and IST (Information Society Technologies) projects, namely the UPGRADE [15], ESTHER [15] and ATLAS [16]. The filters were implemented using monolithic microwave integrated circuit (MMIC) technologies, in order to have a small physical size and integration with other receiver

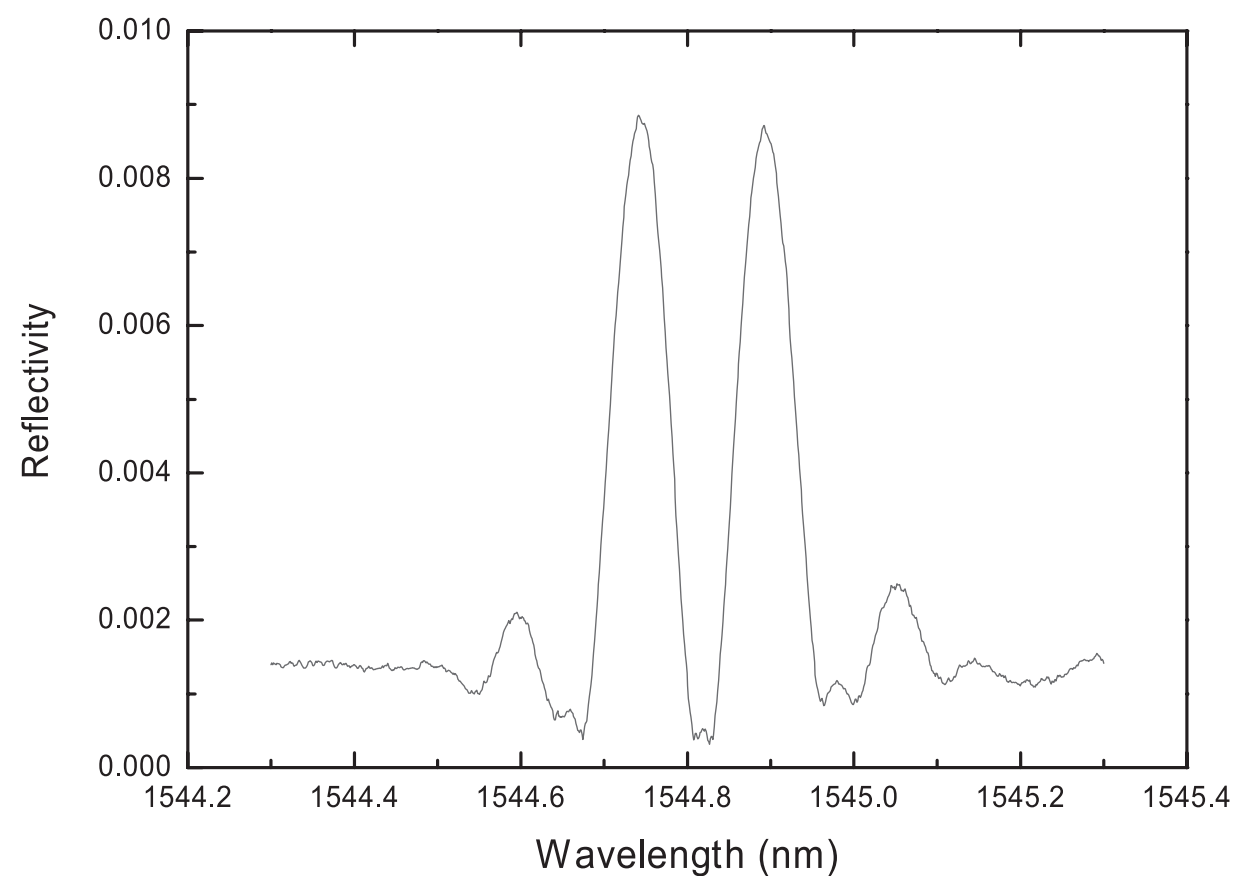

Figure 3. Reflectivity spectrum of a fiber Bragg grating optical cavity. 
components. The electrical tuning facilities of the realized filters allow the minimization of the bit error-rate, for different communication system operating conditions [15].

Presently, IT Aveiro jointly with PTInovação SA and several other international partners integrating the "The Most Transimpedance Highly Efficient Micro\&milimetrewave Optical Smart Transceiver" project consortium, are developing an optical transceiver with dynamic electronic dispersion compensation (EDC) of group velocity dispersion (GVD) and differential group delay (DGD) [17]. The developed EDC systems for $10 \mathrm{Gbit} / \mathrm{s}$ and $40 \mathrm{Gbit} / \mathrm{s}$ are based on a post-feed-forward equalizer (FFE). For a bit error rate of $10^{-9}$, values of $1750 \mathrm{ps} / \mathrm{nm}$ and $104 \mathrm{ps} / \mathrm{nm}$ of dispersion compensation where achieved while mitigating for up to $65 \mathrm{ps}$ and $16 \mathrm{ps}$ of DGD at $10 \mathrm{Gbit} / \mathrm{s}$ and $40 \mathrm{Gbit} / \mathrm{s}$, respectively.

Concerning the subject of signal processing, recently we have started the study of optical resonant cavities, obtained with fiber Bragg gratings. These structures have a wide application range, mainly in the all-in fiber signal processing, and we have proposed them as a fundamental piece in optical clock recovery circuits [18]. In Figure 3 we show the spectrum of an optical cavity designed to have an intra-cavity oscillating frequency of $12.5 \mathrm{GHz}$.

\section{Optical Communication Systems}

The work on optical communication systems is carried out mainly from the point of view of dense wavelength division multiplexing (DWDM) systems, with increasing activity on optical time division multiplexing (OTDM). Some of the areas considered are:

- Line coding, pulse formatting and modulation techniques [19] for the optical channel, such as optical solitons, highly dispersive pulses, single side-band signaling, and DPSK-related formats

- Optical and electrical strategies for static and dynamic compensation of group velocity dispersion (GVD) [20, 21] and polarization mode dispersion (PMD) [22]

- Development of analytical and simulation tools for comprehensive system performance assessment and optimization [23, 24]

- Optical 3R regeneration [18] and transparent/regenerative wavelength conversion

- All-optical performance monitoring [25].

Some of the issues related to the design and optimization of DWDM systems, namely optimization of optical filtering, signaling formats, dispersion compensation strategies in presence of nonlinear transmission-induced degradation, and the impact of noise and crosstalk in optical networks, are described in detail in the accompanying paper [24].

On the subject of modulation techniques for the optical channel, we are also studying the optical single sideband (OSSB) transmission techniques, for upgrading the capacity of the existing fiber networks. We are also considering the viability of OSSB transmission for the forthcoming generation networks, with experimental demonstration in a test bed. A new synthesis approach was used to design FIR filters, with a close approximation to the Hilbert transform, for AM-SSB generation of wide-band signals. The new method can reduce the Vestigial Side Band up to 59\% when compared with that obtained with a conventional FIR filter design method [26].

To improve the performance of the OSSB systems electrical equalization was investigated. The results obtained demonstrated that an electrical equalizer based on a 4th order transversal filter with coefficients calculated for zero forcing criteria, improves the transmission distance up to $215 \mathrm{~km}$ at a BER of $10^{-9}$. This result represents an increment 
of fiber span by $55 \mathrm{~km}$, when compared to the maximum OSSB transmission distance without equalization [27].

High-bandwidth transimpedance GaAs MMIC receivers suitable for $10 \mathrm{Gbit} / \mathrm{s}$ and $40 \mathrm{Gbit} / \mathrm{s}$ data transmission rates were also designed and characterized [28, 29]. The designed circuits were implemented using a MMIC PH15 process from United Monolithic Semiconductors (UMS). A modular semi-analytical tool was also developed, for the noise characterization of distributed optical preamplifiers for very high-capacity optical transmission [30, 31] and a $45 \mathrm{GHz}$ distributed MMIC amplifier implemented [32]. Due to the high potential of the OSSB transmission, a new project ETOBLU has been recently funded by a telecommunication equipment manufacturer (Siemens S.A.) to study the applicability and implementation of optical single sideband transmission for the next generation telecommunication equipment at 10 and $40 \mathrm{Gbit} / \mathrm{s}$.

On the subject of group velocity dispersion (GVD) we observe that the increasing transmission rates and link lengths of DWDM systems, realized over standard singlemode fiber (SMF), demand a better knowledge of the temperature impact on the systems. Since the chromatic dispersion of the deployed optical fibers depends on temperature, it is important to know the relation between chromatic dispersion and chromatic dispersion slope with changes in the temperature, in order enable the full dispersion compensation over the wide temperature ranges normally encountered in practical systems [33]. We have demonstrated that change in temperature induces variations of the dispersion and dispersion slope on the dispersion compensation fibers (DCF). Which have the opposite signal of those changes occurring in SMF fiber. Thus temperature controllable DCF fibers could be used as first and second order dispersion compensators for the SMF fibers [21].

Still in the area of fiber dispersion, we have been studying some fundamental issues related with polarization-mode dispersion (PMD) in high-speed fiber-optic transmission systems. We pay particular attention to issues such as the PMD-induced pulse broadening, PMD measurement and emulation, as well as PMD compensation. Details about these studies carried at the Institute of Telecommunications are presented in another full-length paper included in this issue [22].

In relation to the optical performance monitoring (OPM), our main motivation is the implementation of dense wavelength division multiplexing (DWDM) systems. This is one of the elected techniques for further upgrading the capacity of the existing point-topoint transmission links, for achieving the required increase in transmission throughput. The next evolution step will be the migration to all-optical networks, implying that signals with several modulation formats, bit rates, and protocols may be present at any given localization. Current networks are managed, protected, and monitored after previous conversion from the optical to the digital domain; however, the concept of all-optical networks requires the utilization of monitoring techniques also in the optical domain, introducing the OPM concept. During the last years we have worked in the optimization, implementation, and test of OPM techniques.

In our method, OPM is achieved through the determination of the statistical characteristics of an asynchronously detected optical signal and by using the asynchronous Q-factor to monitor the signal degradation [34]. Figure 4 displays an eye diagram of a synchronously detected $2.5 \mathrm{Gbits} / \mathrm{s}$ signal (left) and the respective asynchronously sampled diagram (right). The latter does not need a synchronized clock, a feature that leads to a simpler and transparent implementation. However, a histogram obtained from such asynchronous samples corresponds to an approximation of the monitored signal's probability density function. 


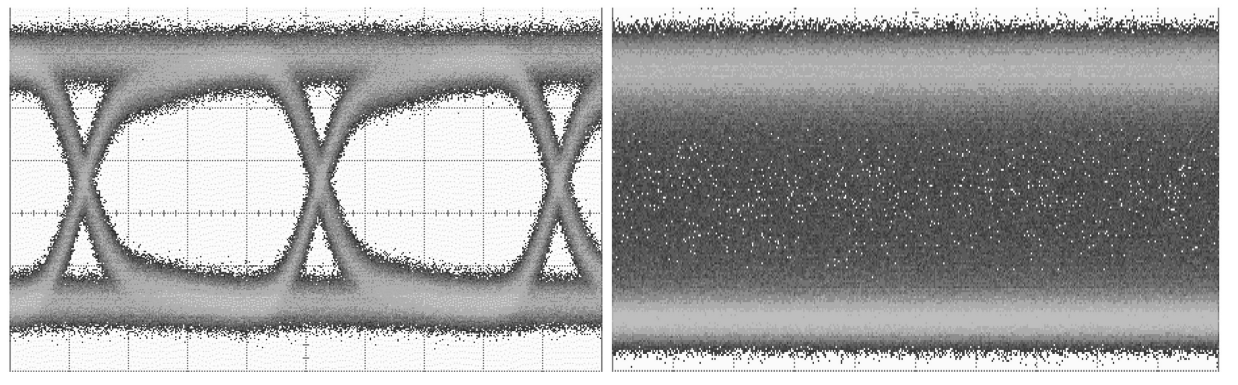

Figure 4. Synchronous eye diagram for a $2.5 \mathrm{Gbit} / \mathrm{s}$ optical signal (left) and the corresponding asynchronous eye diagram (right).

The asynchronous Q-factor for a $2.5 \mathrm{Gbit} / \mathrm{s}$ signal obtained by this method reflects the signal degradation due to the accumulation of noise, waveform distortion due to chromatic dispersion, nonlinear effects, and intersymbol interference [25, 35]. This method was extended in order to characterize $40 \mathrm{Gbit} / \mathrm{s}$ optical signals [36]. Recently we have studied the limitations imposed by non-ideal sampling systems in asynchronous sampling based Q-factor monitoring techniques [37].

\section{Optical Networking}

In relation to optical networking, the following topics are being pursued:

- Studies on topology

- Performance analysis and design of optical transport networks taking into account the constraints due to the physical layer (optical fiber, optical components) and the upper layer protocols to be serviced

- Automatic switched optical networks: planning, routing, protection and restoration

- Optical code division multiple access (OCDMA) [38]

- Optical packet/burst switching.

As regards the restrictions placed on network design by various physical layer limitations, we note that the maximum number of nodes that a network with a physical ring topology can support depends on physical restrictions, on the node structure as well as on the protection strategy. In our studies we have assumed that the nodes are optical adddrop multiplexers (OADMs) constructed using arrayed-waveguide gratings and the main physical restrictions are the crosstalk from OADMs, fiber and component losses, optical amplifier gain saturation and optical amplifier noise accumulation. We have considered SPRings (shared protection rings) and assumed that the protection operates at the multiplexer section (OMS) level. To estimate the maximum number of nodes in the presence of physical restrictions a transmission model was developed that uses the bit error ratio (BER) as a quality metric and considers uniform traffic demand between nodes [39]. Applying this model to a 2-fiber OMS-SPRings shows that for a bit rate of $2.5 \mathrm{~Gb} / \mathrm{s}$, the ring cannot support more than 9 nodes if a BER of $10^{-12}$ is required, if very strict crosstalk requirements are imposed [40]. This number can increase using a 4-fiber OMS-SPRing as can be concluded from Figure 5, where the OADM input power is represented as a function of the number of nodes for the normal state and protection state. Actually, this figure shows that a network with 15 nodes is feasible provide that the OADM crosstalk is less than $-35 \mathrm{~dB}$ and the amplifier saturation power is larger than $15 \mathrm{dBm}$. 


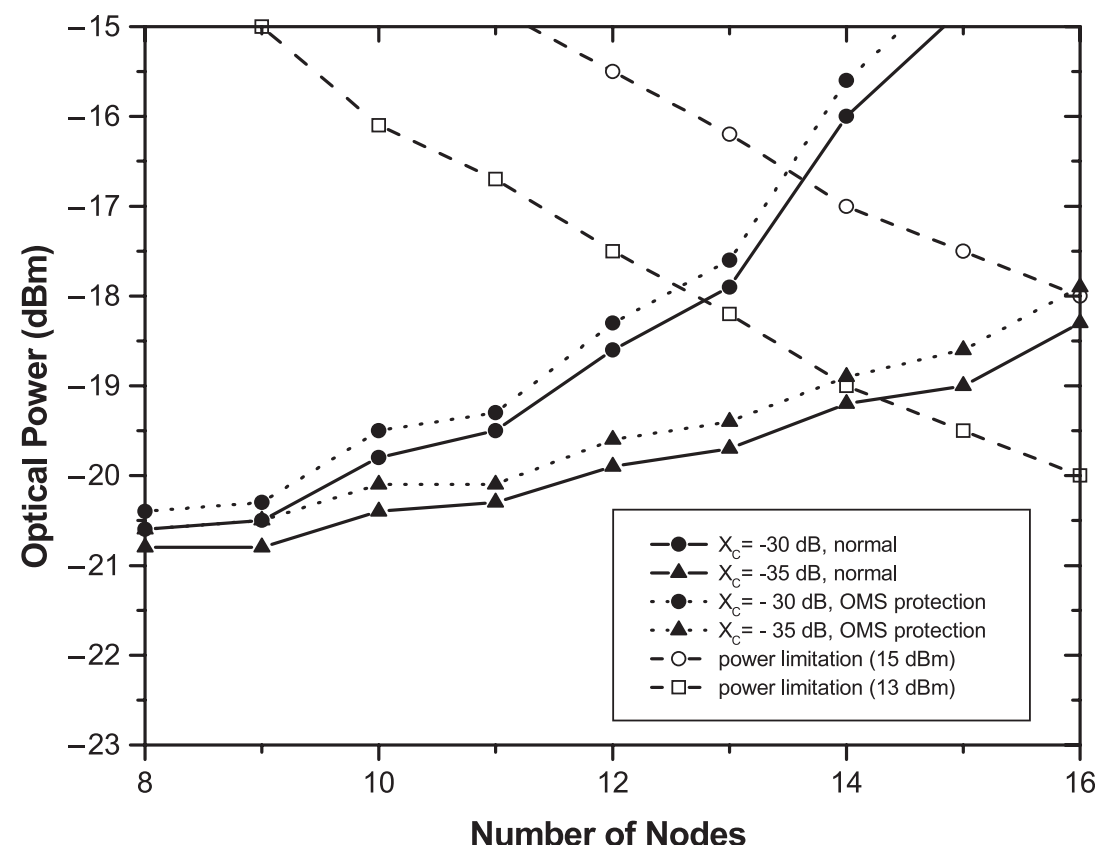

Figure 5. Input power (@BER $=10^{-12}$ ) versus the number of nodes for a 4-fiber OMS SPRing, operating in normal state (solid lines), or in the protection state (dotted lines) with crosstalk $\left(X_{c}\right)$ as a parameter. Dashed lines correspond to the maximum available power per two values of the amplifier saturation power.

The work realized in optical networking comprises also the study of techniques and algorithms for efficient and reliable optical transport network planning. The network planning problem is often decomposed in various sub-problems such as topology design, routing and wavelength assignment (RWA), and protection strategy. In the topology design stage it is common to map a real-world network on a multi-ring structure in order to achieve a high degree of resilience and consider a dual node interconnection between rings. In our studies we treat the problem of multi-ring generation and dual node interconnection as a whole, rather than separate problems, as they are usually analyzed, and use the simulated annealing algorithm to optimize the network cost. The results obtained show a significant $20 \%$ savings in the network cost in comparison with the conventional approach. The RWA algorithm that we have proposed for multi-ring networks uses an exact routing algorithm to handle the traffic inside the rings and a hyper-ring concept to deal with inter-ring traffic, while the wavelength assignment problem is treated as a graph coloring problem [41]. Using this algorithm a range of protection schemes can be compared. It is shown, namely, that by sharing the protection capacity, as the case of SPRings (shared protection rings), the number of required wavelengths can be reduced up to 50\% in comparison with the dedicated strategy used in DPRings (dedicated protection rings), assuming a random traffic demand between nodes. The architectures that we have considered for providing the dual-node interconnection are based on the drop-and-continue concept, imported from SDH, and the sections between the primary node and the secondary node can be implemented by using the service capacity or the protection capacity. 
On the subject of packet switching, the AOLS (all-optical label swapping) technique has been proposed, in order to increase the network efficiency and capacity of optical switching networks. In this technique packet reading and routing are carried out directly in the optical domain, avoiding the electronic payload processing. Recently, the orthogonal modulation technique has also been suggested. In this case, the label is modulated in FSK (frequency shift keying) format, while the IP payload is modulated in ASK (amplitude shift keying). The proposed method has the advantage of providing a simple way to label extraction and encoding processes. We have experimentally and theoretically studied the transmission characteristics for a hybrid modulation format (FSK/ASK), optimizing the emitter characteristics, such as extinction ration, and the chromatic dispersion management [20].

Another aspect that we are investigating at IT relates to the cost impact of scalability of optical transport networks. In the current economic environment, network transport service providers must balance the initial capital outlay for setting up the network, with the lowest total cost of ownership over the network lifecycle, which depends on the flexibility as regards network scalability. We are considering the merits of two different optical network architectures and compare the cost of each alternative as network traffic grows. Therefore, we mainly address the scalability problem (in terms of transmission capacity) of the optical transport network. The issue of scalability concerns whether the networks can scale with the traffic growth and be expandable at reasonable costs.

The first approach is based on optical transmission and electrical switching equipment, such as SDH digital cross connects (DXC), with capacity for switching time multiplexed containers. The optical signal is converted to the electrical domain in each node, switched and converted back into the optical domain before feeding an optical transmission system again. The alternative approach is based on optical transmission and switching equipment, namely optical cross connects (OXC), with capacity for switching individual optical wavelengths.

We consider realistic scenarios and equipment costs [42]. First, we start with a moderate loaded traffic matrix, and afterwards we increase the traffic. Although, the specific results depend greatly on the chosen scenario, there are some general results that become clear after considering several and distinct scenarios. The major point is that the choice for the technology to use must be a balanced compromise on the initial cost budget and the network scalability. The best solution tends to be a hybrid solution due to the fact that both technologies can coexist in the same network. We note that some network nodes are mostly passing through nodes, in which case the possibility of using optical switching equipment can be evaluated. Otherwise, generally, electrical switching would be cheaper. The network efficiency (occupancy) will be better with electrical switching due to the implicit flexibility of the time multiplexed technique. The full equipment occupancy leads to cheaper unit cost, but increases the scalability price and reduces the possibility of accommodation of sporadic traffic increase. Beyond the spare bandwidth, which facilitates scalability, optical switching could be used for extra protection, restoration, and network control schemes.

In IT, research has also been carried out with optical code division multiple access (OCDMA), for applications in LANs, and considering non-coherent detection. Such systems have significant advantages in terms of the complexity required to build the transmitters and receivers, but because they are based on intensity modulation, the signature codes are restricted to unipolar $(0,1)$ sequences. This non-negative nature of the signals prevents the use of the well-studied bipolar codes commonly used in radio communications and provided the motivation for the development of unipolar pseudo-orthogonal 
codes. The number of available codes is, however, rather low and they are clearly inferior in terms of auto-correlation or cross-correlation properties to the well-designed bipolar codes such as Gold or Kasami sequences, thus putting limits on the maximum number of simultaneous users. To allow the use of bipolar codes with incoherent detection, solutions essentially based on complementary keying were proposed either for time or spectral coding. The work at IT [43] has dealt with balanced complementary receivers considering both synchronous and quasi-synchronous networks, and the objective was to find bipolar codes with good properties when used in a quasi-synchronous system employing balanced receivers. Sequences based on Gold codes, tolerant to jitter, were derived and proposed for LANs where the jitter tolerance allows the implementation of network synchronization without excessive complexity.

In the area of optical packet/burst switching (OBS) we have focused on the study of 1) network topologies for OBS mesh networks and 2) on the performance of signaling protocols for OBS networks. In order to carry out this research work, an object-oriented simulator for OBS networks has been developed [44]. Almost all of the published work in this area is based on linear or ring topologies or on well-known and already installed WDM mesh topologies such as the NSFNET and the European Optical Network (EON). Since burst loss is a key issue in OBS networks and several topologies with the same nodal degree may lead to different network performances, network topology may become an important feature for network planning using OBS technology. The research work in this area is focused on the network performance taking into account the following mesh topologies [45-49]: chordal rings with nodal degrees between 3 and 6, meshtorus, the NSFNET with 14-node, the NSFNET with 16 nodes, the ARPANET with 20 nodes, and the European Optical Network (EON) with 19 nodes. For comparison purposes bi-directional ring topologies are also considered. Figure 6 shows the nodal degree gain, as a function of the nodal degree, due to the increase of the nodal degree from

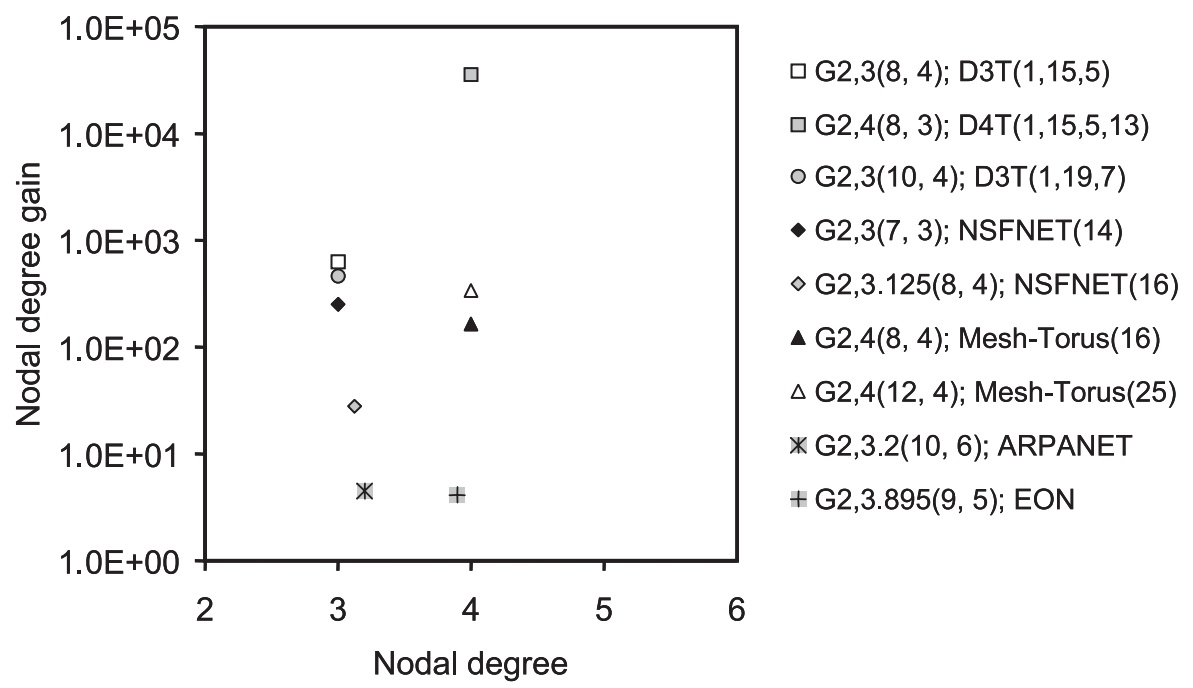

Figure 6. Nodal degree gain in the last hop of each topology, as a function of the nodal degree, due to the increase of the nodal degree from $2[\mathrm{D} 2 \mathrm{~T}(1,15)]$ to: $3[\mathrm{D} 3 \mathrm{~T}(1,15,5)$ and $\mathrm{D} 3 \mathrm{~T}(1,19,7)]$, 3.125 (NSFNET), 3.895 (EON), 4 [D4T(1, 15, 5, 13) and mesh-torus with 16 and 25 nodes], and 5 [D5T(1, 15, 7, 3, 9)], for JIT signaling protocol; Number of data channels per link: 64 . 
2 [D2T(1,15)] to: 3 [D3T(1, 15, 5) and D3T(1, 19, 7)], 3.125 (NSFNET), 3.895 (EON), 4 [D4T $(1,15,5,13)$ and mesh-torus with 16 and 25 nodes], and 5 [D5T $(1,15,7,3,9)]$. As may be seen, when the nodal degree increases from 2 to around 3, the largest gain is observed for degree-three chordal rings (slightly less than three orders of magnitude) and the smallest gain is observed for the ARPANET (less than one order of magnitude). When the nodal degree increases from 2 to around 4, the largest gain is observed for degree-four chordal rings (with a nodal degree gain between four and five orders of magnitude) and the smallest gain is observed for the European Optical Network (with a gain less than one order of magnitude). These results clearly show the importance of the way links are connected in OBS networks, since, in these kind of networks, burst loss probability is a key issue [46, 49].

Concerning signaling protocols for OBS networks, most of the published work does not consider several important parameters such as the offset length, the processing time of setup messages, and the configuration time of optical switches, which have significant impact on burst loss. Research activities in this topic are focused on the performance of signaling protocols with one-way reservation schemes taking into account the abovereferenced parameters. The following protocols are being considered: just-in-time (JIT), JumpStart, $\mathrm{JIT}^{+}$, just-enough-time (JET), and Horizon [44, 46, 49].

\section{Conclusions}

In this contribution we have described the main research activities in optical communications at Institute of Telecommunications. Our research considers various aspects of optical communications, namely components and subsystems, communication systems, and networking technologies. Most of the work carried out in these sub-fields aims at contributing to the implementation of all-optical networks, with very high bit rates per wavelength.

\section{References}

1. André, P. S., R. N. Nogueira, A. L. J. Teixeira, M. J. N. Lima, J. F. da Rocha, and J. L. Pinto. 2004. Multi wavelength rational harmonic mode locked source for polarization division multiplexing based on a reflective semiconductor optical amplifier and Bragg grating written in a high birefringent fiber. Laser Physics Letters 1(4):1-4.

2. Melo, M., O. Frazão, A. L. J. Teixeira, L. A. Gomes, J. R. F. da Rocha, and H. M. Salgado. 2003. Tunable L-band erbium-doped fibre ring laser by means of induced cavity loss using a fibre taper. Applied Physics B: Lasers and Optics 77:139-142.

3. Nogueira, R., I. Abe, H. Kalinowski, J. F. da Rocha, and J. L. Pinto. 2002. Precise characterization of fiber Bragg gratings for photonic communications systems. Fourth International Conference on Photonics, Devices and Systems 176-181.

4. Teixeira, A., P. André, M. Lima, J. Pinto, and J. da Rocha. 2004. Applications of highly birefringent fibre Bragg gratings. Sixth International Conference on Transparent Optical Networks.

5. Lima, M. J. N., A. L. J. Teixeira, and J. R. F. da Rocha. 2000. Simultaneous filtering and dispersion compensation in WDM systems using apodised fibre gratings. Electronics Letters $1412-1414$.

6. Teixeira, A. L. J., M. J. N. Lima, and J. R. F. da Rocha. 2000. Effects of gratings center frequency tolerances in WDM optical networks. Thirteenth Annual Meeting of the IEEE Lasers \& Electro-Optics Society, 484-485

7. Lima, M. J. N., A. L. J. Teixeira, J. R. F. da Rocha, O. Frazão, and P. S. B. André. 2003. Comparison of the thermal tuning capability of different types of Bragg grating filters for wavelength division multiplexing applications. SPIE Journal of Optical Engineering 2502-2505. 
8. Abe, I., H. Kalinowski, R. N. Nogueira, J. L. Pinto, and O. Frazão. 2003. Production and characterisation of Bragg gratings written in high birefringence fibre optics. IEE ProceedingsCircuits Devices Syst. 495-500.

9. Andre, P. S., J. L. Pinto, A. J. Teixeira, A. N. Pinto, T. Almeida, F. Morgado, and M. Pousa. 2002. Strictly non-blocking all-optical-cross-connect demonstrator for WDM wavelength path networks. Photonic Network Communications 4:63-72.

10. Nogueira, R. N., A. L. Teixeira, I. Abe, J. F. da Rocha, and J. L. Pinto. 2002. Self-pumped wide range wavelength converter. Fifteenth Annual Meeting of the IEEE Lasers and Electro Optics Society.

11. Nogueira, R. N., A. L. Teixeira, P. André, I. Abe, H. Kalinowsky, J. F. Rocha, and J. L. Pinto. 2003. All-fiber broad band orthogonal pumps wavelength converter. Tenth International Conference on Telecommunications.

12. Teixeira, A., D. Pereira, S. Junior, M. Lima, P. André, R. Nogueira, J. Rocha, and H. Fernandes. 2002. Black box model of erbium-doped fiber amplifiers in $\mathrm{C}$ and $\mathrm{L}$ bands. Eleventh International Conference on Telecommunications.

13. Nogueira, R. N., A. L. J. Teixeira, P. S. André, J. F. da Rocha, and J. L. Pinto. 2002. Wide Tuning Range Self-Generated Orthogonal Pumps Source Based on a Reflective Semiconductor Optical Amplifier. Conference on Lasers and Electro-Optics.

14. Nogueira, R. N., A. L. J. Teixeira, J. R. F. da Rocha, and J. L. Pinto. 2004. Constant Efficiency Broadband Wavelength Converter based on Self-Generated Orthogonal Pumps. Electronics Letters 616-617.

15. Monteiro, P., A. Borjak, J. R. F. da Rocha, J. J. O’Reilly, and I. Darwazeh. 2002. Adjustable Post-Detection Filters for Optically Amplified Soliton Systems. IEICE Transactions on Electronics E85-C:511-518.

16. Monteiro, P. P., M. Violas, R. Ribeiro, and J. F. da Rocha. 2003. Electronic Post-detection Filter for Multigigabit Optical Communication Systems. Eleventh IEEE International Symposium on Electron Devices for Microwave and Optoelectronic Applications, 192-197.

17. Machado, J. E. S., A. N. Pinto, J. M. Santos, P. M. Monteiro, M. Violas, and L. M. R. Teixeira. 2004. Transversal Filter Model for High Bit Rate Optical Channel Equalization. Symposium on Enabling Optical Networks.

18. Curti, F., G. M. T. Beleffi, D. M. Forin, A. Teixeira, R. Nogueira, P. André, A. Fiorelli, A. Reale, and S. Betti. 2005. All-Optical Clock Recovery and Resampling at $10 \mathrm{Gbit} / \mathrm{s}$ for NRZ Data Signals. Submitted to 2005 OSA /IEEE Optical Fiber Conference.

19. Teixeira, A. L. J., R. N. Nogueira, P. S. B. André, M. J. N. Lima, and J. R. F. da Rocha. 2004. Optimized Wavelength Interleaved Radio-over-Fibre System based on Highly Birefringent Fibre Bragg Gratings. Accepted for publication in Electronics Letters.

20. André, P. S., A. T. Teixeira, P. Monteiro, M. J. N. Lima, R. N. Nogueira, J. R. F. Rocha, and J. L. Pinto. 2004. Dispersion compensation in IP-over-DWDM networks employing combined modulation formats. Journal of High Speed Networks.

21. André, P. S., B. Neto, J. L. Pinto, A. L. J. Teixeira, M. J. Lima, and F. da Rocha. 2004. Allocable Dispersion Compensation for Thermal Dispersion Variations in Transmission Fibers. Conference on Laser and Electro Optics.

22. Ferreira, M. F., A. N. Pinto, P. S. André, N. J. Muga, J. E. Machado, R. N. Nogueira, S. V. Latas, M. H. Sousa, and J. F. Rocha. 2005. Polarization Mode Dispersion in High-Speed Optical Communication Systems. Fiber and Integrated Optics 24(3-4):261-285.

23. Pizzinat, A., A. Schiffini, F. Alberti, F. Matera, A. N. Pinto, and P. Almeida. 2002. 40 Gb/s Systems on G.652 Fibers: Comparison Between Periodic and All-at-the-End Dispersion Compensation. IEEE/OSA Journal of Lightwave Technology 20:1673-1679.

24. Cartaxo, A. V. T., J. L. Rebola, N. B. Pavlovic, P. M. A. Charrua, D. D. T. Fonseca, J. A. P. Morgado, R. S. Luís, and M. R. G. Leiria. 2005. Overview of DWDM/ODC project. Fiber and Integrated Optics 24(3-4):353-369.

25. André, P. S., J. L. Pinto, A. L. J. Teixeira, M. J. N. Lima, and J. F. da Rocha. 2002. Bit error rate assessment in DWDM transparent networks using optical performance monitor based in asynchronous sampling. Optical Fiber Communications Conference, 749-750. 
26. Moura, L., and P. Monteiro. 2002. Design method for FIR-based Hilbert transform filters suitable for broadband AM-SSB. Electronics Letters 38:605-606.

27. Maia, T., E. Costa, A. Pinho, R. Ribeiro, P. Monteiro, M. Violas, and J. F. da Rocha. 2003. $10 \mathrm{Gbit} / \mathrm{s}$ Optical Single Sideband Transmission using Zero Forcing Equalization. Third International Conference on Wireless and Optical Communications, 104-109.

28. Madureira, M. A. M., P. M. P. Monteiro, R. L. Aguiar, M. Violas, M. Gloanec, E. Leclerc, and B. Lefebvre. 2003. High Gain GaAs 10 Gbps Receiver with Integrated Bondwire Effects. IEEE International Symposium on Circuits and System.

29. Madureira, M. A. M., P. M. P. Monteiro, R. L. Aguiar, M. Violas, M. Gloanec, E. Leclerc, and B. Lefebvre. 2002. Broadband Optical Receiver for Multigigabit-per-second (40Gb/s) Optical Communications Systems. Tenth IEEE International Symposium on Electron Devices for Microwave and Optoelectronic Applications.

30. Moura, L., P. Monteiro, and I. Darwazeh. 2001. Exact noise analysis of distributed preamplifiers for optical receivers. Microwave and Optical Technology Letters 30:416-418.

31. Moura, L., P. Monteiro, and I. Darwazeh. Generalized noise analysis technique for four-port linear networks. Accepted for publication in IEEE Transactions on Circuits and Systems.

32. Violas, M., P. M. P. Monteiro, M. Gloanec, E. Leclerc, and B. Lefebvre. 2003. 45 GHz Distributed Amplifier with On Chip Drain Resistor Termination. Thirty-third European Microwave Conference, 1099-1102.

33. André, P. S., and A. N. Pinto. 2004. Chromatic Dispersion Fluctuations in Optical Fibers Due to Temperature and Its Effects in High-Speed Optical Communication Systems. Optics Communications.

34. Teixeira, A. L. J., P. S. André, M. Lima, J. F. da Rocha, and J. L. Pinto. 2002. Asynchronous Optical Performance Monitor Techniques for DWDM Optical Networks. Fourth International Conference on Transparent Optical Networks.

35. André, P. S., J. L. Pinto, A. L. J. Teixeira, J. F. da Rocha, T. Almeida, and M. Pousa. 2002. Optical-signal-quality monitor for bit-error-ratio assessment in transparent DWDM networks based on asynchronously sampled amplitude histograms. Optical Society of America Journal of Optical Networking 1:118-128.

36. André, P. S., A. T. Teixeira, M. J. N. Lima, P. Monteiro, R. Luis, and D. Fonseca. 2004. Asynchronous sampled amplitude histogram models for optical performance monitoring in high speed networks. OSA Journal of Optical Networking 3:636-642.

37. Luis, R., P. S. André, A. T. Teixeira, and P. Monteiro. 2004. Performance monitoring in optical networks using asynchronously acquired samples with nonideal sampling systems and intersymbol interference. IEEE/OSA Journal of Lightwave Tech. 22:2452-2459.

38. Nogueira, R. N., P. S. Taluja, A. L. Teixeira, P. André, J. F. da Rocha, and J. L. Pinto. 2003. New Technique for Implementing Multiwavelength Orthogonal Codes for OCDMA using Fiber Bragg Gratings written in High Birefringence Fibers. Sixteenth Annual Meeting of the IEEE Lasers and Electro Optics Society.

39. Pires, J. J. O., M. O’Mahony, N. Parnis, and E. Jones. 1999. Scaling limitations in full-mesh WDM ring networks using arrayed-waveguide grating OADMs. Electronics Letters, 73-75.

40. Pires, J. J. O. 2001. Constraints on the design of 2-fiber bi-directional WDM rings with optical multiplex section protection. 2001 IEEE/LEOS Summer Topical Meetings (Ultra Long Haul DWDM Transmission and Networking), 13-14.

41. Pedro, J. M., P. P. Monteiro, and J. J. Pires. 2004. Comparison of interconnection architectures for WDM shared protection rings. IASTED Conference on Optical Communication Systems and Networks, 423-456.

42. Carvalho, N. J., N. S. C. Correia, A. Pinto, and M. C. R. Medeiros. 2005. Cost Impact in Scalability of Optical Transport Networks-A Case Study. To be presented at Conftele'05.

43. Gameiro, A. 2000. Synchronous and quasisynchronous optical CDMA with balanced complementary receivers. IEE Proceedings-Optoelectronics 147:370-376.

44. Rodrigues, J. J. P. C., N. M. Garcia, M. M. Freire, and P. Lorenz. 2004. Object-Oriented Modeling and Simulation of Optical Burst Switching Networks. IEEE Global Telecommunications Conference: CAMAD Workshop. 
45. Rodrigues, J. J. P. C., M. M. Freire, and P. Lorenz. 2004. Performance Assessment of Optical Burst Switching Ring and Chordal Ring Networks. In Telecommunications Systems. Kluwer Academic Publishers, 133-149.

46. Rodrigues, J. J. P. C., M. M. Freire, and P. Lorenz. 2004. Performance Assessment of Signaling Protocols with One-Way Reservation Schemes for Optical Burst Switched Networks. In EDITOR(S), High-Speed Networks and Multimedia Communications. Springer-Verlag, 821-831.

47. Freire, M. M., J. J. P. C. Rodrigues, and R. M. F. Coelho. 2003. The Role of Network Topologies in the Optical Core of IP-over-WDM Networks with Static Wavelength Routing. In Telecommunications Systems. Kluwer Academic Publishers, 111-122.

48. Freire, M. M., and H. J. A. da Silva. 2002. Assessment of Blocking Performance in Unidirectional and Bi-directional Wavelength Routed Ring Networks. Journal of High Speed Networks 133-140.

49. Rodrigues, J. J. P. C., and M. M. Freire. 2004. Performance Assessment of Signaling Protocols in Optical Burst Switching Mesh Networks. In Hyun-Kook Kahng, Information Networking: Networking Technologies for Broadband and Mobile Networks. Heidelberg, Germany: Springer-Verlag, 750-759.

\section{Biographies}

José R. F. da Rocha was born in Mozambique and received an M.Sc. degree in telecommunication systems and a Ph.D. in electrical engineering, both from the University of Essex, England, in 1980 and 1983, respectively. He is at present a full professor at the University of Aveiro, Portugal. He participates actively in the Telecommunications Institute, a national $R \& D$ non-profit organization, where he is a member of the management committee (Aveiro branch) and national coordinator for the optical communications area. He has coordinated the University of Aveiro and Telecommunications Institute participation in various projects included in the following European Union (EU) R\&D programs in the area of telecommunications: RACE, RACE II, ACTS, and IST. He has published about 170 papers, mainly in international journals and conferences and his present research interests include modulation formats and receiver design for very high-capacity (above $40 \mathrm{Gbit} / \mathrm{sec}$ ) optical communication systems based on linear and nonlinear transmission and WDM optical networks. In the past few years he has acted as a technical auditor, evaluator, and independent observer for the evaluation of projects submitted to various EU R\&D programs. He has also participated in various project evaluation boards set up by the EPSRC (Engineering and Physical Sciences Research Council), United Kingdom. By invitation of the ACTS Management Committee, he participated in the "Expert Groups on Visionary Research in Communications," aiming to create a bridge between the activities carried out in the 4th and the 5th Framework Programs on EU activities in the field of research, technological development, and demonstration.

Adolfo V. T. Cartaxo was born in Montemor-o-Novo, Portugal, in 1962. He received the degree of licenciatura in electrical engineering, an M.Sc. degree in telecommunications and computers, and a Ph.D. in electrical engineering in 1985, 1989, and 1992, respectively, from Instituto Superior Técnico (IST), Lisbon Technical University. In 1985, he joined the Department of Electrical and Computers Engineering of IST. In 1992, he became an assistant professor and he was promoted to associate professor in January 2002. Over these years, he has lectured several courses on telecommunications. He joined the Optical Communications Group of Lisbon Pole of Institute for Telecommunications as a researcher in 1992 . He is now a senior researcher conducting research on dense wavelength division multiplexed systems and networks. He has authored more than 25 journal publications as well as more than 50 international conference papers. He has been leader of the IST participation in three projects of the European Union programs on 
R\&D in the optical communications area and two national projects. In the past few years, he has acted as a technical auditor and evaluator for projects included in several European Union R\&D programs. He has served as a reviewer for several publications in the area of optical communications. He is a senior member of the IEEE Laser and Electro-Optics Society. His main present research interests include fiber optic communication systems and networks.

Henrique J. A. da Silva was born in Luanda, Angola, on November 21, 1953. He received the degree of licentiate in electrical engineering in 1977, from the University of Coimbra, Portugal, and a Ph.D. in communication systems engineering from the University of Wales, United Kingdom, in 1988. He was a research officer at the Department of Electronics and Telecommunications of the University of Aveiro, Portugal, from 1978 to 1982. In 1983 he joined the Department of Electrical and Computer Engineering at the University of Coimbra, where he is now an associate professor. He has been the leader of the Optical Communications Group of the Institute of Telecommunications at Coimbra, Portugal, since 1992. His main research interests include optical communication systems and mobile communications. He is a member of the IEEE Communications Society, IEEE Lasers and Electro-optics Society, and the American Society for Engineering Education.

João de Lemos Pinto has a degree in electrotechnical engineering from the University of Porto. In 2000 he became a full professor of the Department of Physics of the University of Aveiro, Portugal, where he has been lecturing many different theoretical and practical courses, namely applied optics, optoelectronics, and holography. He is presently leading the research group "Optics and Lasers" of the Physics Department of the University of Aveiro and the "Electronic and Optoelectronic Components" research area of the Institute of Telecommunications-Pole of Aveiro. His present research interests include optical communications, Bragg gratings systems, optical image processing, and holography.

António Teixeira was born in 1970 in S. Pedro do Sul, Portugal. He received his degree in engineering in electronics and telecommunications (1994) and Ph.D. (1999) in electrical engineering from the University of Aveiro, Portugal. He is presently an assistant professor at the same university and has been a researcher at the Instituto de Telecomunicações. His present research interests are all-optical networks components, subsystems, and related impairments. Dr. Teixeira is a member of IEEE and OSA.

Atílio Gameiro received his licenciatura (five-year course) and his Ph.D. from the University of Aveiro in 1985 and 1993, respectively. He is currently a professor in the Department of Electronics and Telecommunications of the University of Aveiro, and a researcher at the Instituto de Telecomunicações-Pólo de Aveiro, where he is head of group. His main interests lie in signal processing techniques for digital communications and communication protocols. Within this research line he has done work for optical and mobile communications, either at the theoretical and experimental level, and has published over 100 technical papers in international journals and conferences. His current research activities involve space-time-frequency algorithms for the broadband component of $4 \mathrm{G}$ systems and joint design of layers 1 and 2.

Paulo P. Monteiro was born in Coimbra, Portugal. He received the diploma and doctoral degrees in electronics and telecommunications from the University of Aveiro and an M.Sc. (Eng) degree from the University of Wales, U.K. He is head of research of optical networks at Siemens S.A. and assistant professor at the University of Aveiro, where he has been teaching courses in telecommunications and computer science. He is also a research scientist at the Instituto de Telecomunicações. His main research interests include 
high-speed circuit design and signal processing for gigabit-per-second optical communications systems. Participation in several projects of the European Union programs on R\&D in the telecommunications area; namely, RACE (R1051), RACE II (R2011), ACTS (AC063-ESTHER, AC045-UPGRADE, AC049-SPEED), and IST (1999-10626-ATLAS). At the ATLAS project, he was responsible for the workplace "Electronic and Optoelectronic Devices." He is currently participating at the IST European project MUFINS (Multi-Functional Integrated Arrays of Interferometric Switches) where he is responsible for the workplace "Exploitation and Dissemination." He has published more than 70 research papers in journals and conferences.

João O. Pires is an assistant professor at Instituto Superior Técnico where he is teaching optical communications systems and telecommunication networks. He has worked on a number of European projects such as RACE 1051, RACE 2028 (MWTN), and ACTS 066 (OPEN) and has more than 30 technical publications. His research interest is in optical transport networks.

Rui F. S. Ribeiro was born in Oporto, Portugal, in 1966. He graduated in electronics and telecommunications from the University of Aveiro, Portugal, in 1989. He received a Ph.D. from the University of Aveiro in 1996, where he is a staff member. He has been reviewer of the IEEE Photonics Technology Letters. His main research interests are coherent optical communications, signal processing for high speed communications, and simulations of digital communication systems.

Manuel A. Violas received a degree in electronics and telecommunications engineering from the University of Aveiro, Portugal, in 1982, an M.Sc. from the University of Coimbra, Portugal, 1988, and a Ph.D. in electrical engineering from the University of Aveiro in 1999. Since 1982, he has been with the Department of Electronics and Telecommunications at the University of Aveiro, Portugal. His current research interests include microwave and RF circuit design, microwave circuit/system simulation, and electrical signal processing for very high-speed lightwave communications systems.

Armando Nolasco Pinto was born in Oliveira do Bairro, Portugal, in 1971. He graduated in electronic and telecommunications engineering from the University of Aveiro, Portugal, in 1994, and received a Ph.D. in electrical engineering from the University of Aveiro in 1999. At present, he is an assistant professor at the electrical and telecommunications department of the University of Aveiro, and a researcher at the Institute of Telecommunications, Aveiro, Portugal. His main research interests focus on optical communication systems, mainly in nonlinear, polarization- and temperature-dependent effects in fibers optics. He has written more than 60 scientific journal and conference publications. He is a member of the Optical Society of America (OSA) of the Institute of Electrical and Electronics Engineers (IEEE).

Mário José Neves de Lima was born in Lourosa, Portugal, in 1972. He graduated in electronic and telecommunications engineering from the University of Aveiro, Portugal, in 1994, received an M.Sc. degree in telecommunication systems, and a Ph.D. in electrical engineering, both from the University of Aveiro in 1998 and 2003, respectively. At present, he is an invited assistant professor at the electrical and telecommunications department of the University of Aveiro, and a researcher at the Institute of Telecommunications, Aveiro, Portugal. His main research interests focus on optical communication systems, mainly in optical filtering and fiber Bragg gratings. He is a member of the Optical Society of America (OSA) and of the Institute of Electrical and Electronics Engineers (IEEE).

Paulo Sérgio de Brito André was born in Luanda, Angola, in April 1971. He received a physics engineering degree in 1996 and a Ph.D. in physics in 2002, both 
from the Universidade de Aveiro, Portugal. In the same year he joined the Instituto de Telecomunicações-Aveiro as researcher. He is also assistant professor at the University of Aveiro, teaching physics. His current research interests include the study and simulation of optoelectronics components, fiber Bragg gratings, transparent performance monitoring, multi-wavelength optical communications systems, and networks. Paulo André is member of the Portuguese Physics Society (SPF) and of the Institute of Electrical and Electronics Engineers (IEEE).

Mário Marques Freire was born in Seia, Portugal, on March 21, 1969. He received the degree of licentiate in electrical engineering and an M.Sc. degree in systems and automation in 1992 and 1994, respectively, from the University of Coimbra, Portugal. He received a Ph.D. in electrical engineering from the University of Beira Interior, Portugal, in 2000. Presently, he is an assistant professor at the Department of Informatics of the University of Beira Interior, Covilhã, Portugal, where he is the head of the department and the director of the M.Sc. course in informatics engineering. His main research interests include high-speed networks and Internet technologies. He has been the editor of 2 books and has authored or co-authored around 90 papers in international refereed journals and conferences. He served as a technical program committee member for several IEEE and IASTED conferences. He was the general chair of IEEE HSNMC'2003 and a general co-chair of ECUMN'2004, and he is the TPC Chair of ICN'2005. He is also a member of the editorial review board of the International Journal of Business Data Communications and Networking. He is a licensed professional engineer by the Order of Engineers-Informatics College (Portugal) and he is a member of IEEE Computer Society, IEEE Communications Society, the ACM SIGCOMM, and the Internet Society.

Carlos Alberto Ferreira Fernandes was born in Luanda-Angola, and received a $\mathrm{Ph}$.D. degree in electrical and computer engineering from the Instituto Superior Técnico, Lisbon Technical University, in 1988. He is currently an assistant professor in the Department of Electrical and Computer Engineering at Instituto Superior Técnico, Lisbon, Portugal, and he is a researcher at the Institute of Telecommunications, Lisbon, Portugal. His present research interests are the simulation and characterization of optoelectronic devices and materials. He is a member of the Portuguese Society of Professional Engineers.

Jorge T. Pereira was born in Pero Soares, Guarda, Portugal, and received an M.Sc. and a Ph.D. in electrical engineering from the University of Rhode Island, in 1984 and 1987, respectively. He is currently an associate professor in the Department of Computer and Electrical Engineering at Instituto Superior Técnico, Lisbon, Portugal, and he is a researcher at the Institute of Telecommunications, Lisbon, Portugal. His present research interests are the simulation and characterization of optoelectronic devices and materials. $\mathrm{He}$ is a member of the Portuguese Society of Professional Engineers and of the New York Academy of Sciences.

José Marcelino Pousa was born in Bragança, Portugal, and received a degree in applied physics on optics and electronics, University of Porto, 1984, and an M.Sc. in organization and information systems, 2002, University of Évora. He has worked for the main Portuguese operator since 1986 in the former R\&D labs CET, currently PT Inovação. For 16 years he developed activity in the area of development of optical systems, mainly for the access network, and network strategic planning. He participated in a number of internal and European projects, RACE, IST, and EURESCOM, in the access network and optical communications area, both in PT Inovação and IT (Instituto de telecomunicações). He has assumed management functions as leader of a group for 
the promotion and diffusion of knowledge in PT Inovação, and he is a member of the Management Committee in the IT (Aveiro branch) and Direction of Instituto de Telecomunicações.

José Miguel Santos graduated in electronics and telecommunications engineering from the University of Aveiro (1985) and earned an M.Sc. in sender-chooser dynamic and distributed restoration for telecommunication networks from the University of Aveiro (1998). He has worked for Centro de Estudos de Telecomunicações of Aveiro for since 1991 in transmission network developments and at the global operator GTS Network Services in Brussels for 2 years (since 1998) in the network strategy and planning division where was responsible for the network architecture of the Flag Atlantic1 transatlantic submarine cable. Since 2000 he has worked in PT Inovação Aveiro in the area of the access networks.

Maria Teresa Almeida was born in Portugal in February 1962. She received her degree in physics applied to optics and electronics from the Porto University in 1986. From 1987 to 1989 she worked at LIP-Lisbon, with a fellowship from JNICT, having integrated during this period DELPHI team at CERN. Since 1989 she works in the R\&D company within Portugal Telecom Group, Portugal Telecom Inovação, the former CET, as a researcher. Within PTIn she developed expertise in DWDM networking technology. Her activities include lecturing of company internal courses, test and assessment of DWDM equipment, responsibility of internal projects, co-operation with IT-Aveiro, and participation in several European projects, EC funded (COBRA, PHOTON, HARMONICS, and currently MUSE and NoE E-NEXT) and EURESCOM (P615, P709, P917, BOBAN, and P1012, Fashion). She is presently working in the Technology Evaluation and Selection Department of PTIn. She has authored and co-authored more than 20 publications.

Rogério Nunes Nogueira was born in Aveiro, Portugal, in 1976. He received a physics engineering degree in 1998 from the University of Aveiro. In the same year he joined the Instituto de Telecomunicações as a researcher in the field of optical communications. He is now finishing his Ph.D. research program in fiber Bragg gratings. His current research interests are fiber Bragg gratings, multi-wavelength optical devices and systems, optoelectronic devices, and optical sensors. Rogério Nogueira is a member of the Portuguese Physics Society (SPF) and of the Institute of Electrical and Electronics Engineers (IEEE).

José E. S. Machado graduated in electrical and computer engineering from the University of Porto (2002), Porto-Portugal, accomplishing is final graduation project in polymer optical fibers multiplexing techniques within COBRA Research Institute, Eindhoven University of Technology, Eindhoven, The Netherlands. After a short traineeship in core network department of VIP Snaga Komunikacije, Zagreb-Croatia, he joined PTInovação SA (2003), carrying out most of his work at Instituto de Telecomunicações, Aveiro labs. In the framework of project "The Most Transimpedance Highly Efficient Micro\&milimetrewave Optical Smart Transceiver" his main interests are within dynamic dispersion compensation for multi-gigabit optical networks. 